\title{
Sites naturels sacrés et conservation des ressources marines et côtières en milieu traditionnel diola (Sénégal)
}

Exemple du Bliss-Kassa et de l'aire du patrimoine autochtone et communautaire de Mangagoulack

Sacred natural sites and the conservation of marine and coastal resources in the traditional Jola environment: Example of Bliss-Kassa and the Indigenous people's and community conserved territories and areas in Senegal

\section{Claudette Soumbane Diatta, Malick Diouf, Charlotte Karibuhoye et Amadou Abdoul Sow}

\section{(2) OpenEdition}

Journals

Édition électronique

URL : http://journals.openedition.org/ethnoecologie/2900

DOI : $10.4000 /$ ethnoecologie.2900

ISSN : 2267-2419

Éditeur

Laboratoire Eco-anthropologie et Ethnobiologie

Référence électronique

Claudette Soumbane Diatta, Malick Diouf, Charlotte Karibuhoye et Amadou Abdoul Sow, « Sites naturels sacrés et conservation des ressources marines et côtières en milieu traditionnel diola (Sénégal) », Revue d'ethnoécologie [En ligne], 11 | 2017, mis en ligne le 03 juillet 2017, consulté le 01 mai 2019. URL : http://journals.openedition.org/ethnoecologie/2900 ; DOI : 10.4000/ ethnoecologie. 2900

Ce document a été généré automatiquement le 1 mai 2019.

Revue d'ethnoécologie est mis à disposition selon les termes de la licence Creative Commons Attribution - Pas d'Utilisation Commerciale - Pas de Modification 4.0 International. 


\section{Sites naturels sacrés et conservation des ressources marines et côtières en milieu traditionnel diola (Sénégal)}

Exemple du Bliss-Kassa et de l'aire du patrimoine autochtone et communautaire de Mangagoulack

Sacred natural sites and the conservation of marine and coastal resources in the traditional Jola environment: Example of Bliss-Kassa and the Indigenous people's and community conserved territories and areas in Senegal

Claudette Soumbane Diatta, Malick Diouf, Charlotte Karibuhoye et Amadou Abdoul Sow

\section{Introduction}

1 Les sites naturels sacrés (SNS) sont avant tout des lieux culturels et cultuels, mais ils apportent également des bénéfices pour la conservation. De nos jours, ils sont de plus en plus reconnus comme des moyens efficaces pour contribuer à la préservation des ressources naturelles. Pourtant, on observe la désacralisation de certains de ces sites, du fait de l'adoption des religions dites révélées qui banalisent ces pratiques animistes d'une part, et des mutations en cours dans la société traditionnelle d'autre part. Très peu d'études ont été réalisées sur les SNS côtiers et marins en Afrique de l'Ouest en général et au Sénégal en particulier, pour faire connaître leur contribution à la conservation de la biodiversité. La méconnaissance de la contribution des SNS à la conservation de la biodiversité fait que ces derniers sont peu valorisés. Les travaux réalisés sur la question concernent en général les sites naturels terrestres tels que les forêts et les bosquets sacrés. Ces travaux ont traité des avantages de ces lieux reliés à un système de croyances, 
mais aussi à une organisation sociale qui renvoie à un certain contrôle des ressources naturelles.

Les recherches sur le sujet ont commencé à être développées dans les années 1960 avec notamment les travaux de ADAMS sous l'égide de l'UNESCO (Petitjean et al. 2009). Mais, l'intérêt des études a connu un véritable essor en 1997, avec l'UNESCO dans le projet intitulé « Sacred sites, Cultural integrity and Biological Diversity 》 (Juhé-Beaulaton 2008). Depuis, les études sur ce thème se sont multipliées. En Afrique, notamment au Bénin, Burkina Faso et Togo, Juhé-Beaulaton (2006) a réalisé une étude sur les enjeux politiques, économiques et sociaux des SNS. À travers cette étude l'auteur met en exergue l'importance tant culturelle, écologique, économique que politique des forêts sacrées dans ces régions. Au Maroc, Taïqui et al. (2009) ont abordé la question sur la conservation des SNS et leur incompatibilité avec le développement socio-économique. Cette étude est axée sur l'analyse des SNS, leur spécificité, leur densité et leur niveau de conservation en relation avec les caractéristiques socio-économiques.

3 Au Sénégal, particulièrement en Basse Casamance dans le département d'Oussouye, Badiane a mené en 2006 une étude sur les pratiques culturelles et la gestion des ressources forestières des Diolas d'Oussouye. Dans son étude, il a tenté de démontrer comment les ressources naturelles forestières ont pu être préservées grâce aux méthodes de gestions traditionnelles usitées par ce peuple. Chabi-Yaouré (2009) s'est surtout intéressée aux pratiques et connaissances traditionnelles de gestion des ressources naturelles marines et côtières dans les localités de Pointe Saint Georges et du Petit Kassa ${ }^{1}$. À notre connaissance les études sur les sites sacrés marins et côtiers existants dans ce pays sont celle de Ndiaye \& Diagne (2004) sur les sites sacrés de la Presqu'île de Cap-Vert, celle de Fall et al. (2010) et celle de Oyono et al. (2012) initiées par la FIBA dans le cadre de l'étude sous-régionale d'identification et de caractérisation des SNS côtiers et marins en Afrique de l'Ouest. Dans le cadre de ces deux dernières études, la recherche au Sénégal a concerné les sites de Sangomar situés dans la Réserve de Biosphère du delta du Saloum et du Petit Kassa en Basse Casamance au sein des villages insulaires de Haer et Bakassouk. Dans ses travaux, Diatta (2012) a mis en évidence le rôle des sites naturels sacrés marins et côtiers du Petit Kassa et de l'Aire du Patrimoine Autochtone et Communautaire de Mangagoulack. Nous pouvons également noter quelques travaux remarquables menés en Basse Casamance. Il s'agit entre autres des travaux de Thomas $(1958,1959)$, de Pélissier (1966), de Journet (1976, 1979, 1998), de Palmeri (1990), de Diedhiou (2011).

En Basse Casamance, notamment dans le Bliss-Kassa et dans la zone de l'aire du patrimoine autochtone et communautaire (APAC) de Mangagoulack, les traditions animistes régissent toujours la vie sociale des Diolas ${ }^{2}$. Toutefois, les deux zones se distinguent par des dimensions spirituelles animistes plus marquées dans le Bliss-Kassa. Les éléments naturels contribuent de manière importante à l'équilibre social des populations locales. Ceci se traduit entre autres par la sacralisation de plusieurs espaces dans le milieu marin et côtier. Ces espaces servent de refuge aux totems et génies, mais représentent aussi des lieux de culte et de rituels où prévalent des règles d'accès et d'usage qui varient selon les SNS. Ces endroits font le lien entre l'homme, la nature, la culture, les valeurs spirituelles et religieuses. Djoghlaf (2006) les qualifie de trait d'union entre la nature, la culture, les valeurs spirituelles et éthiques.

5 Nous visons ici à contribuer à une meilleure connaissance du fonctionnement des SNS côtiers et marins, mais aussi à mettre en lumière leur contribution à la conservation 
durable des ressources de ces milieux au Sénégal en général et en Casamance en particulier.

\section{Bliss-Kassa et l'Aire du Patrimoine Autochtone et Communautaire (APAC) de Mangagoulack}

6 Les sites du Bliss-Kassa et l'APAC de Mangagoulack sont situés en Basse-Casamance dans le Sud du Sénégal. Ils appartiennent à la région administrative de Ziguinchor, département de Bignona et sont respectivement localisés dans les Communes de Kafountine et de Mangagoulack.

7 Le Bliss-Kassa regroupe les villages de Bakassouk, Hitou, Niomoune, Haer et Diogué. Il est situé dans la partie basse de la région du fleuve Casamance entre $12^{\circ} 44^{\prime}$ et $16^{\circ} 44^{\prime}$ de latitude Nord et $12^{\circ} 34^{\prime}$ et $16^{\circ} 46^{\prime}$ de longitude Ouest. Il est limité à l'ouest par l'océan Atlantique, à l'est par la commune de Mlomp (zone de Blouf), au nord par les îles Bliss et au sud par le fleuve Casamance. L'APAC de Mangagoulack encore appelée Kawawana couvre les villages de Tendouck, Boutégol, Mangagoulack, Elana, Diatock, Boutème, Bodé et Affignam. Elle est située sur la rive nord de la ria de Casamance également dans le Blouf. Il se positionne entre $12^{\circ} 40^{\prime}$ et $16^{\circ} 25^{\prime}$ de latitude Nord et $12^{\circ} 37^{\prime}$ et $16^{\circ} 32^{\prime}$ de longitude Ouest entre la Gambie au nord, la Guinée Bissau au sud, l'océan Atlantique à l'ouest et la rivière Gouloumbou à l'est. Les villages de l'étude sont Bakassouk, Hitou, Niomoune et les huit villages qui composent l'APAC (Figure 1).

8 L'hydrographie de la zone est marquée par la présence du fleuve Casamance et de ses nombreux bras ou bolongs. Dans le Bliss-Kassa, on compte trois principaux bolongs : Ounambène, Ouniomouneye et Asséléghène, ainsi qu'une multitude de petits bolongs interconnectés qui se jettent en général dans le marigot de Diouloulou ou dans le fleuve Casamance. Dans l'APAC de Mangagoulack, les principaux bolongs sont le bolong de Thionck Essyl à l'ouest plus ou moins parallèle au marigot de Diouloulou, et le bolong de Tendouck, qui est relié à celui de Thionck Essyl par le bolong de Badiapour au nord. Il existe d'autres bolongs très connus dans cette zone, tels que Ecobe encore apelé KilingKiling à l'est et le Mitij, en confluence avec ceux de Kadiountang et de Thionck Essyl.

9 Le climat de type sud soudanien côtier (Sagna 2007), est caractérisé par l'alternance d'une saison sèche (octobre à mai) et d'une saison humide (juin à septembre). La température est relativement douce. La moyenne annuelle sur la période de 1970-2014 est de $28^{\circ} \mathrm{C}$ avec un minimum et un maximum annuel moyen respectifs de $21,5^{\circ} \mathrm{C}$ et $34,5^{\circ} \mathrm{C}$. La pluviométrie moyenne annuelle est de 1234,3 mm (période de 1970-2014) à la station de Ziguinchor située à $12^{\circ} 33^{\prime}$ de latitude nord et $16^{\circ} 16^{\prime}$ de longitude ouest à une altitude de $25 \mathrm{~m}$.

$10 \mathrm{Au}$ recensement général de la population, de l'habitat, de l'agriculture et de l'élevage (RGPHAE) de 2013 du Sénégal, la population s'élevait à 8477 habitants dans la commune de Mangagoulack et à 31340 habitants dans celle de Kafountine. Les Diolas constituent l'ethnie majoritaire soit $93 \%$ des effectifs dans la commune de Mangagoulack et $87 \%$ dans celle de Kafountine (Groupe OCC 2010a, Groupe OCC 2010b). D'autres ethnies sont présentes à savoir les Mandings, les Peulhs, les Ouolofs, etc. La population diola est en majorité animiste et chrétienne dans le Bliss-Kassa (Dieme 2008), et musulmane pour $65 \%$ des habitants dans la commune de Mangagoulack (PLD Mangagoulack 2010). La majorité 
de la population s'active dans le secteur primaire (agriculture, élevage, exploitation des ressources forestières).

\section{Méthodes}

11 Un questionnaire proposé aux ménages, a permis d'obtenir des informations sur :

- La relation sites naturels sacrés-population, afin de rendre compte de la présence de SNS dans la zone d'étude, la raison de leur sacralité, les règles et mesures de leur gestion.

- La place des sites naturels sacrés dans la gestion des ressources naturelles afin d'appréhender le rôle des SNS dans la conservation des ressources naturelles. Nous nous sommes également intéressés aux menaces auxquelles ils sont exposés.

12 L'échantillonnage des ménages est tiré des données du recensement général de la population et de l'habitat de 2002 (DPS 2002) pour le Bliss-Kassa et du recensement local effectué par la communauté en 2006 pour l'APAC de Mangagoulack (Borrini-Feyerabend 2009). Au total, 153 ménages ont été interrogés. Pour compléter les informations relatives à l'histoire et au fonctionnement des sites sacrés nous avons eu recours à des groupes de discussions ciblés, souvent appelés « focus group ». Dans ces groupes hommes et femmes étaient interrogés séparément. Ainsi, nous avons mené à bien des entretiens groupés de 10 à plus de 30 personnes parfois. Quelques entretiens informels sans questionnaires ont concerné les personnes âgées, les sages et les chefs coutumiers.

13 Nos séjours sur le terrain d'enquête nous ont également permis d'effectuer d'une part des observations directes sur des faits, des pratiques, des objets, etc., pertinents, et en rapport avec la problématique de recherche et d'autre part d'effectuer des visites dans les SNS respectifs.

Figure 1 : Répartition par zone de la population interviewée

\begin{tabular}{|c|c|c|c|}
\hline \multirow{2}{*}{ Localités } & \multicolumn{2}{|c}{ Groupes de discussion ciblés } \\
\hline Bliss-Kassa & 26 & 7 & Nombre de groupes \\
\hline Mangagoulack & 127 & 14 & Entre 10 et 30 \\
\hline & & 70 Entre 7 et 20 \\
\hline
\end{tabular}

Source : Direction de la Prévision et de la Statistique 2002 \& Conseil Rural de Mangagoulack 2006 in Borrini-Feyerabend 2009

\section{Résultats}

Les sites naturels sacrés côtiers et marins suscitent de plus en plus d'intérêt au regard du rôle qu'ils peuvent jouer dans la protection des ressources naturelles et de la biodiversité en général. Leur existence remonterait à une date très lointaine qui coïnciderait probablement avec l'installation des premiers occupants des villages de l'étude. D'après Snyder (1973), la région Basse-Casamançaise serait habitée depuis 300 A.D. Les enquêtes de terrain ont permis de répertorier au total 51 sites naturels sacrés dans les deux zones, avec une prédominance des sites dans le Bliss-Kassa, soit $55 \%$ de l'effectif contre $45 \%$ dans la zone de l'APAC de Mangagoulack. Cette prédominance de sites sacrés dans le Bliss-Kassa s'explique par le fait que malgré les mutations intervenues récemment (modernité, religions monothéistes), cette communauté a su conserver ses traditions. 
Figure 2 : Localisation des sites naturels sacrés étudiés dans le Bliss-Kassa et de l'Aire du Patrimoine Autochtone et Communautaire de Mangagoulack

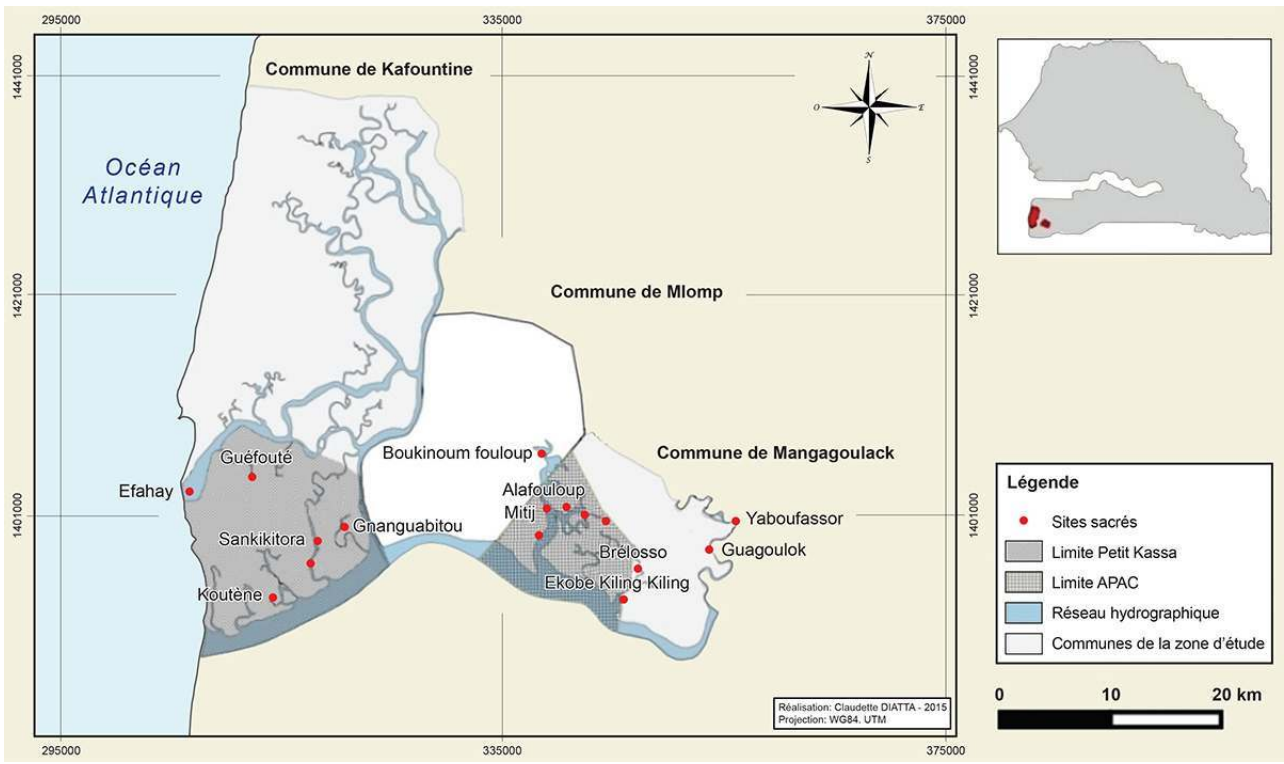

Réalisation : C.S. DIATTA (Source : DTGC)

Les résultats présentés concernent les SNS d'Efahay yata Salindia, Guéfouté, Sankikitora, Koutène, Gnangabitou, Diahébène situés dans le Bliss-Kassa et Mitij, Boukinoum fouloup, Alafouloup, Koutéwol, Foumor sithégour, Barotor, Brélosso, Ecobe (plus connu sous le nom de Kiling-kiling), Guagoulok et Yaboufassor ${ }^{3}$ localisés dans l'APAC de Mangagoulack. Le choix de ces SNS a été fait suivant la fréquence avec laquelle ils ont été cités et aussi en fonction de leurs spécificités. Toutefois, plus de sites sacrés naturels marins ont été étudiés dans l'espace communautaire de Mangagoulack, du fait de la diversité des types de SNS rencontrés dans cette localité.

\section{Typologie des sites naturels sacrés du Bliss-Kassa et de l'Aire du Patrimoine Autochtone et Communautaire de Mangagoulack}

16 Les sites naturels sacrés rencontrés dans les villages de Bakassouk, de Hitou et de Niomoune au Bliss-Kassa sont pour l'essentiel des lieux de résidences des totems, alors que dans l'APAC, ils sont de cinq types. Il s'agit de :

17 - lieux de résidence de totems,

- sites abritant un fétiche (boekin en Diola),

- sites où résident des génies ou "djinns ",

- sites habités par un génie ou djinn et où un fétiche a été placé pour protéger les ressources de la zone, sites de culte.

Chaque type de SNS se distingue par ses propres caractéristiques. Les SNS qui abritent les totems servent d'habitat. Le totem (au singulier éwoum, au pluriel siwoum en diola) est l'espèce animale symbolisant le double de chaque individu d'une famille ou d'un groupe social. Il marque les liens de consanguinité ou d'alliance qui lient l'individu ou le lignage à certains animaux. Dans le système totémique, chaque naissance dans la famille correspondrait à une naissance chez le totem et si un malheur (maladie ou mort) atteint 
le totem, son double humain est aussi touché. Les totems sont considérés comme tabous et il est formellement interdit de les blesser encore moins de les tuer.

Les SNS qui abritent un fétiche ont pour vocation la conservation d'une ou plusieurs ressources naturelles. En effet dans ces SNS, l'extraction des ressources naturelles est soit interdite (Mitij, Kiling-kiling), soit réglementée (Guagoulok). Ces sites ont été créés par les populations pour freiner la dégradation des ressources halieutiques. Cependant, Guagoulok participe aussi à la protection des rizières. Notons que les fétiches sont des entités surnaturelles qui facilitent la relation entre les hommes et la nature mais surtout avec le seigneur du ciel, et de la pluie ou ata emit (Palmeri 1990). Ils sont craints et très respectés dans la société diola et constituent un moyen de dissuasion efficace usité pour faire respecter les règles instaurées.

Les sites servant d'habitacle aux génies ou « djinns » sont également vus comme sacrés et très craints par les populations locales. Du fait des mystères qui les entourent et la crainte profonde qu'ils suscitent chez les populations, ces endroits sont paisibles. Ils représentent ainsi des milieux privilégiés de ponte, de refuge et de nurserie pour certaines espèces aquatiques de la zone. Parmi ces endroits on peut citer Badiapour ou personne n'ose s'aventurer pour pêcher. Des sites comme Gouhi abrite des plantes médicinales auxquelles seul un homme d'une certaine sagesse et investi d'un pouvoir surnaturel lui permettant de communiquer avec le génie tutélaire a le droit d'accéder pour prélever les feuilles ou racines permettant de soigner des malades.

Le seul SNS qui abrite à la fois un génie et un fétiche, à savoir Kiling-kiling ${ }^{4}$ a pour vocation de protéger les ressources halieutiques. Ce site est la demeure d'un puissant génie, qui considérait les poissons comme "ses enfants ». Il n'interdisait pas la pêche, mais obligeait les pêcheurs à prélever la ressource de manière raisonnable. L'on raconte que les jours où le génie ne voulait pas de pêche dans le site, il se métamorphosait en pêcheur et se mettait à l'entrée du bolong en faisant semblant de pêcher. Au pêcheur qui arrivait et demandait si la pêche était bonne, pour le décourager, il répondait qu'il ne prenait les poissons qu'un par un, kiling-kiling. En cas de pêche excessive, il s'adressait directement au pêcheur en le sommant d'arrêter. L'entêtement du pêcheur conduisait à la destruction du filet, à sa perte ou au chavirement de la pirogue. Aujourd'hui, la crainte que suscitait ce lieu s'est dissipée. Plusieurs raisons l'expliquent; des pratiques surnaturelles ou mystiques de certains pêcheurs auraient contribué à rendre le génie moins redoutable ; l'introduction de pirogues motorisées aurait également poussé le génie à se retirer dans les profondeurs. Pour ces raisons, Kiling-kiling fut soumis à une très forte pression (surpêche, coupe anarchique du bois de mangrove). La raréfaction du poisson a alors amené les populations locales à protéger le bolong par un fétiche, dans le but de restaurer les fonctions écologiques (lieu de reproduction et nurserie) que remplissait ce site d'antan.

Le site de culte (Barotor) est lié à des pratiques de prières et d'offrandes. El Hadji Omar Foutiyou Tall ${ }^{5} s^{\prime} y$ serait arrêté lors de son voyage en Casamance pour assouvir sa soif. Ce site, qui fut autrefois un lieu de pèlerinage, n'est aujourd'hui fréquenté que par les autochtones pour solliciter la grâce divine et déposer leurs offrandes (des pièces d'argent, du lait caillé, etc.). Barotor ne fait pas l'objet d'une exploitation à proprement parler. Il sert de zone de reproduction pour les carpes et d'abreuvoir pour les animaux.

La majorité des SNS étudiés (75\%) sont des habitacles de totems. Le requin apparaît comme l'espèce totémique la plus fréquemment rencontrée. Parmi les autres espèces 
totémiques rencontrées, on compte la baleine, le crocodile, l'hippopotame, le lamantin. (Voir annexe 1).

Figure 3 : Typologie et gestion des sites naturels sacrés (SNS)

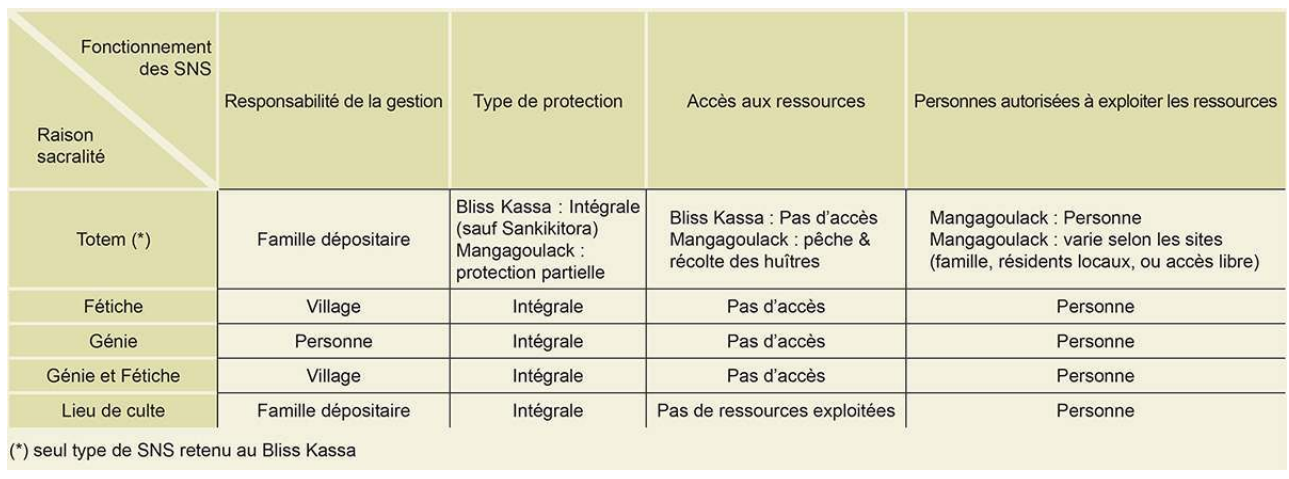

Source : Enquêtes de terrain 2011

\section{Systèmes traditionnels de gouvernance et de protection des sites naturels sacrés}

Les espaces déclarés sacrés sont régis par de nombreuses règles et stratégies endogènes de protection qui en interdisent l'exploitation pour certains, ou la réglementent pour d'autres. Les stratégies de protection des SNS identifiés diffèrent selon les localités. Dans le Bliss-Kassa par exemple, aucun prélèvement (coupe de bois, cueillette des huitres, pêche) n'est autorisé dans les SNS (excepté le site Sankikitora où la récolte des coquillages est permise). En effet, selon les croyances locales, ces endroits doivent être paisibles pour le bien-être des totems. Dès lors y pêcher ou récolter des huîtres pourrait perturber la quiétude du lieu et par conséquent celle des totems. On pense aussi qu'en récoltant les huîtres elles peuvent tomber et les blesser. En y pêchant également, on pourrait capturer un totem qui se serait éloigné du groupe. Ceci pourrait être dangereux pour ce dernier, car il peut être blessé voire même mourir. Au niveau de la zone de Mangagoulack, l'approche est plus flexible; les règles d'accès aux sites vont de l'interdiction totale à l'accès libre, en passant par l'accès restreint aux familles dépositaires ou aux résidents locaux. Des mesures de gestion de l'APAC sont mises en place, notamment en matière de zonage, pratiques de pêche sélective, usage d'engins réglementaires, pour protéger les totems et réglementer le prélèvement dans les sites.

\section{Sites naturels sacrés situés hors des aires protégées officiellement reconnues}

Les sites naturels sacrés situés hors des aires protégées sont gérés de manière traditionnelle comme par le passé. Les détenteurs de pouvoirs surnaturels procèdent par des dissuasions mystiques et des sanctions sont prévues pour ceux qui transgressent les règles.

Cependant, cette intervention humaine ne limite que dans les SNS à totem, les sites de culte et ceux qui abritent un fétiche. Les sites hantés réputés être des habitacles de génie n'entrent pas dans le lot des sites sacrés marins gérés par les populations. Ils sont naturellement protégés par le génie qui y a élit domicile. En cas d'infraction, c'est le génie 
lui-même inflige la correction au fautif soit en détruisant son filet, soit en faisant chavirer son embarcation.

Dans le Bliss-Kassa, le mode de gouvernance interdit l'exploitation des ressources naturelles dans la majorité des sites naturels sacrés. La coupe de bois, la cueillette des huîtres, la pêche sont formellement interdites dans l'ensemble des SNS étudiés de cette zone, à l'exception de Sankikitora où seule la récolte des coquillages est autorisée. Celle-ci se fait toutefois suivant un certain nombre de règles. Il existe également dans cette localité du Bliss-Kassa, des sites où la consommation de certaines ressources halieutiques notamment les huîtres, n'est permise qu'aux hommes âgés ou ceux ayant effectué certains rites initiatiques. Dans le cas de cette étude, seul le site naturel sacré de Guéfouté est concerné par cet interdit. La récolte et la consommation d'huîtres provenant des sites de Kalandaga, Eténia, Djatahounouho, Djihindou et Kassia sont interdites à ceux qui n'assistent pas aux cérémonies qui s'y tiennent (Chabi-Yaouré 2009). Les femmes n'ont point le droit de consommer les huîtres de ces endroits.

Les SNS abritant un fétiche sont frappés d'interdits à respecter sous peine d'être sanctionné. Dans le cas des SNS à totem, la famille dépositaire qui procède au préalable à des avertissements avant que le fétiche n'infliger au fautif la sanction méritée. L'avertissement est fait mystiquement ou de façon verbale par les membres de la famille offensée. En cas de récidive, le coupable est sanctionné par un malheur (blessure ou maladie) qui peut conduire à la mort. Ces malédictions peuvent n'épargner ni la famille, ni la descendance du fautif. Les interdits auxquels sont soumis ces sites s'expliquent par le fait qu'ils abritent des totems. Chez les Diolas comme pour beaucoup de populations africaines (Gadou 2003), le totem est vu comme le parent consanguin de l'homme. Cette relation parentale se retrouve également chez d'autres peuples sénégalais comme les Lébous, les Sérères, etc. Chez les Lébous, Dumez \& Ka (2000) soulignent que certaines lignées possèdent un animal-totem qu'il leur est interdit de tuer parce qu'il est lié soit à un ancêtre, soit à un génie. Certains éléments de l'environnement associés aux matriclans des Sérères Ndut sont considérés comme des parents et désignés par le terme de mboko (Dupire 1991).

Ces considérations vis-vis de l'espèce totémique font apparaître le lien particulièrement étroit qui unit la personne et son double animal. En effet, tout ce qui arrive au totem se répercute automatiquement sur son double (la personne correspondante). Si le totem est blessé, cette blessure va se répercuter sur son double humain. La mort du totem entraîne fatalement celle de son double. Ce lien intrinsèque est mis en exergue par Journet-Diallo (1998) dans son étude sur «la théorie joola du double animal ». L'auteure écrit que chez les diolas :

« le double animal est l'objectivation discontinue d'une part essentielle du moi. Sa destinée est entièrement liée à celle de son homologue humain... Si le double est blessé, son homologue humain subira une même atteinte au même endroit du corps".

30 C'est donc pour veiller à leur tranquillité et ne point porter atteinte à leur vie que les lieux qui leur servent d'habitat et eux-mêmes sont entourés d'interdits qu'il faut respecter sous peine d'être châtié. Dans cette logique, si un pêcheur fait exprès de pêcher à l'endroit interdit, on peut, par des faits mystiques, lui faire prendre dans son filet ou son hameçon le totem de son propre enfant ou celui d'un membre de sa famille. De ce fait, par crainte de voir sa progéniture atteinte d'un mal ou mourir des suites de cette faute on évite de pêcher dans ces lieux. Cet aspect craintif par rapport aux interdits liés aux 
totems est évoqué par Journet-Diallo (1998) qui souligne que chez les Felup ${ }^{6}$ «c'est plutôt la crainte de se faire du mal, voire de tuer un de ses enfants qui prédomine ».

Les SNS font l'objet de cérémonies et de rituels (sacrifices, libations, prières, etc.) qui se tiennent sur la terre ferme devant le fétiche des familles respectives ou celui du village. Ces rituels peuvent parfois s'exécuter sur le site même ; c'est le cas des SNS de Guagoulok et de Boukinoum fouloup. Les rituels sont réalisés pour demander des grâces au génie protecteur mais aussi pour protéger les sites, les personnes, leurs occupants (totems) et les ressources des nuisances dont ils sont victimes. Ils se tiennent également à l'occasion de l'arrivée d'un nouveau-né, dans le but de le présenter au génie protecteur du site sacré et aux ancêtres défunts de la famille. Ces rituels sont exécutés par le chef spirituel, à n'importe quel moment de l'année (dès que le besoin se fait sentir) pour les sites encore respectés. Ce guide spirituel de sexe masculin dans la majorité des cas, assure la gestion du site. Il est choisi dans la famille. Dans le cas des SNS communs à tout le village, le prêtre est choisi parmi les membres de la communauté ou au sein d'une famille désignée à cet effet.

Figure 4 : Vue d'un fétiche à Hitou

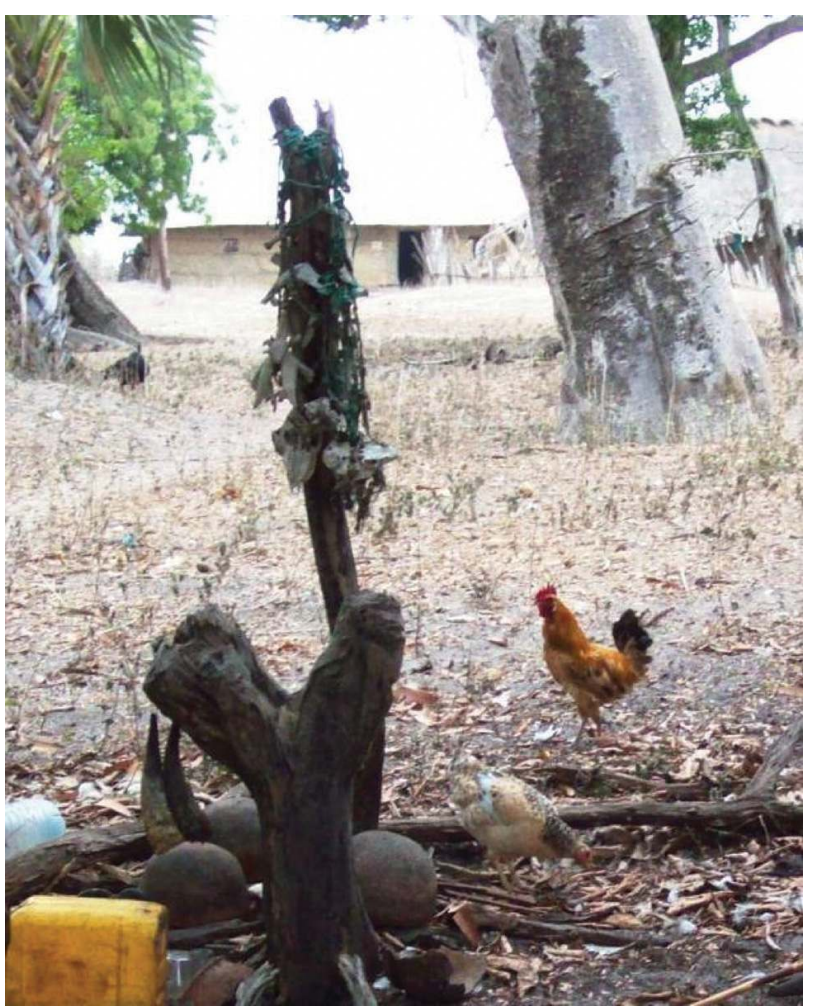

(c) C. Diatta 2011 
Figure 5 : Arbre fétiche du site naturel sacré de Guagoulok

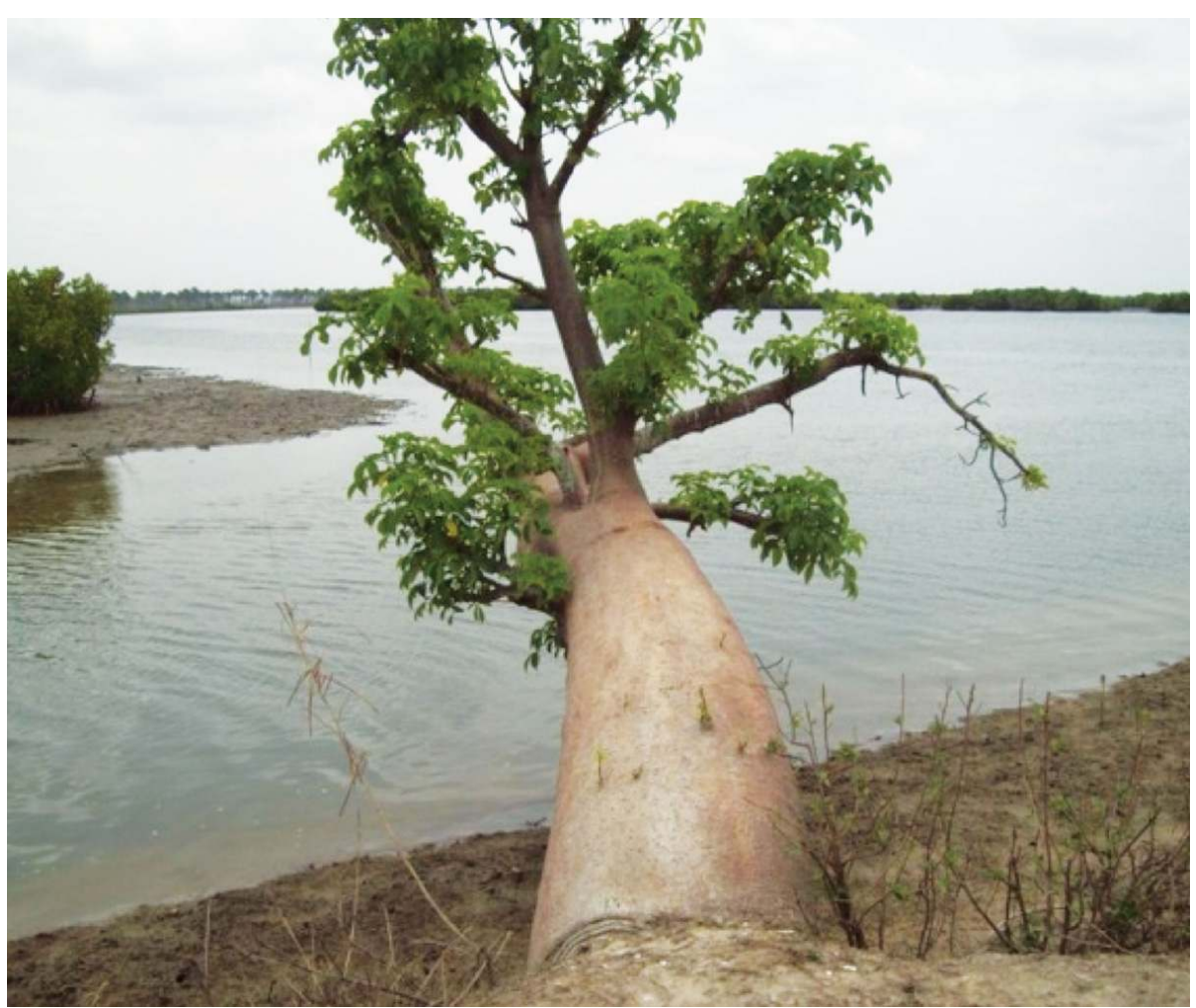

(c) C. Diatta 2011

Dans certains SNS la présence des femmes n'est pas permise lors des cérémonies de libation et d'offrandes. C'est le cas de Guagoulok où seuls les hommes ont le droit d'assister aux cérémonies réalisées pour protéger les rizières, le bolong et les ressources dont ils regorgent. L'objectif de ces cérémonies est de demander un meilleur rendement pour les récoltes et une profusion de poissons. Par ailleurs, hommes et femmes peuvent se retrouver dans un même culte. C'est le cas du site de Eloenkin ${ }^{7}$ qui associe partiellement les femmes à son culte mais les incluent dans sa protection (Badiane 2012). Certains sites sont la chasse gardée des femmes : c'est le cas des Ehuña ${ }^{8}$ dans le Kassa.

\section{Sites naturels sacrés intégrés dans une aire protégée officiellement reconnue}

Les sites naturels sacrés (SNS) ont longtemps été et sont toujours en partie entretenus et gérés à l'écart des systèmes nationaux de conservation par les cultures locales. Aujourd'hui, à cette gestion traditionnelle s'est joint le système moderne, dans le cas de création d'aires protégées (AP) officiellement reconnues par la juridiction nationale, lesquelles sont mises en place afin d'assurer la protection des sites et la pérennisation des ressources.

Dans notre zone d'étude, on dénombre deux sites protégés reconnus officiellement: la Réserve Ornithologique du Kalissaye (ROK) et l'Aire du Patrimoine Autochtone et Communautaire de Mangagoulack. La ROK fut créée en 1978 vu l'importance des colonies d'oiseaux répertoriées dans cette zone fluvio-marine. Bien qu'abritant le SNS de Efahay yata Salindia ${ }^{9}$, sa vocation principale est la préservation de l'avifaune. Le cadre réglementaire interdit toutes formes d'exploitation dans les limites de la réserve. Les 
activités humaines sous toutes leurs formes sont interdites, sauf autorisation spéciale à des fins scientifiques ou de prophylaxie humaine ou animale délivrée par le Ministère en charge des Parcs Nationaux (article $3 \mathrm{du}$ décret présidentiel portant sur le classement du site de Kalissaye en réserve). L'APAC de Mangagoulack (Kawawana) créée en 2010, à l'initiative de l'association des pêcheurs de la commune rurale de Mangagoulack a comme objectif de préserver les ressources halieutiques et de préserver le patrimoine culturel et les pratiques traditionnelles. Dans le zonage de l'APAC, le bolong de Mitij (SNS où se pratiquaient traditionnellement des cérémonies destinées à maintenir la paix sociale et de bons rapports entre l'homme et la nature) est intégralement protégé. Aucune forme d'exploitation n'est autorisée dans le site. Il en est de même pour le SNS d'Alafouloup qui, avant l'avènement de l'APAC, était uniquement exploité par la famille dépositaire. Aujourd'hui, il est inaccessible (fermé) à cause de sa localisation dans la zone de préservation intégrale (bolong de Mitij). De nos jours, certains sites comme Brélosso et Foumor sithégour n'existent plus que de nom. Le caractère sacré de ces sites a parfois disparu car les totems auraient été déplacés vers des zones plus tranquilles du fait de la forte pression anthropique à laquelle ils ont été soumis. Les SNS marins bénéficient depuis la création de l'APAC d'une protection officielle.

Il convient de signaler qu'il existe une différence fondamentale entre ces deux sites protégés, du fait de leur statut légal, du processus de leur création et du type de gouvernance. Au Sénégal, les réserves sont mises en place sur l'initiative des autorités étatiques et comme les parcs nationaux et les forêts classées, elles sont créées par décret présidentiel. Leur gestion est assurée par l'organisme de tutelle désigné. La création de l'APAC émane d'une volonté locale et requiert l'approbation de l'autorité décentralisée (Kante 2012). En effet, la loi sur la décentralisation (Loi 96-06 et Loi 96-07 du 22 mars 1996) a permis de responsabiliser les collectivités locales en matière de gestion de l'environnement et contrairement aux réserves, une APAC est gouvernée par la communauté locale concernée (Borrini-Feyerabend et al. 2009).

Les aires protégées officielles de la zone d'étude abritent chacune un ou plusieurs SNS. Cependant leur objectif principal n'est pas de les protéger, mais plutôt de préserver une ou plusieurs ressources. Toutefois, du fait de ce statut, les SNS situés à l'intérieur de ces AP sont en principe protégés des menaces auxquelles ils sont exposés (désacralisation, pressions anthropiques de plus en plus intenses et perturbation des conditions naturelles). Néanmoins il faut noter que dans ces cas, le système traditionnel de gestion des SNS n'est pas toujours préservé ou valorisé (exemple de Efahay, Alafouloup). Les AP dans lesquelles ces SNS sont localisés, bien que favorisant leur préservation, peuvent dans certains cas entraver le maintien des activités traditionnelles liées aux sites. En ce qui concerne le SNS de Efahay, la création de la ROK n'a pas eu directement beaucoup d'impact puisque les rituels sont traditionnellement exécutés sur la terre ferme. Par contre pour Alafouloup, les rituels pouvaient se faire soit sur le site (milieu marin) ou à distance, autour d'un puits sur terre ferme suivant les besoins. Depuis la mise en place de l'APAC, les rituels ne se tiennent plus que sur la terre ferme; la famille dépositaire n'a plus accès au site. La reconnaissance, le maintien et l'intégration des systèmes et des institutions traditionnels chargés de la gestion des SNS dans le cadre légal dit moderne (indépendamment du type de gouvernance de l'AP) constituent un des principaux enjeux pour ces sites. 


\section{État de conservation des SNS étudiés}

37 Les SNS étudiés montrent un état de conservation différent d'un site à l'autre. Une mangrove relativement dégradée annonce le bord des bolongs où se localisent les SNS de Koutène, Gnangabitou, Boukinoum fouloup, Foumor sithégour, Brélosso et Ecobe. L'état de dégradation reste significatif au niveau de Sankikitora, de Diahébène, de Guagoulok et de Yaboufassor. Malgré une homogénéité apparente, cette dégradation de la mangrove est beaucoup plus avancée au niveau de Guagoulok et de Yaboufassor. La dégradation du couvert végétal dans les sites de Guagoulok et de Yaboufassor serait due en grande partie aux eaux rejetées par le barrage d'Affignam, entraînant l'acidification et la pollution des bolongs. Par contre dans les SNS de Sankikitora et de Diahébène, la dégradation du couvert végétal est due à la coupe de bois abusive et au déficit pluviométrique. C'est au niveau des SNS de Guéfouté, de Mitij, de Alafouloup et de Koutéwol qu'est observée une végétation de mangrove bien conservée. Les SNS de Efahay yata Salindia et de Barotor correspondent respectivement à une bande de sable et une mare à proximité du bolong. Ils sont naturellement dénudés, c'est-à-dire dépourvus de végétation.

Les sites naturels sacrés subissent des pressions de diverses natures parmi lesquelles on note les mauvaises pratiques de pêche, l'exploitation abusive du bois vert de la mangrove, les changements climatiques et les conséquences d'aménagements tels que les barrages. Dans les SNS étudiés, la végétation est relativement bien préservée. Ainsi, $25 \%$ des SNS identifiés ont une végétation bien conservée, $37,5 \%$ présentent une végétation relativement bien conservée, $25 \%$ sont dégradés et $12,5 \%$ sont dépourvus de végétation (annexe 2).

Les SNS ne sont pas à l'abri des menaces de désacralisation et par conséquent de disparition. Toutefois, il convient de dire que la protection officielle des SNS permet sans doute de réduire les menaces dont ils font aujourd'hui l'objet. Par exemple dans la commune de Mangagoulack où les pratiques animistes ne sont plus aussi vivaces que par le passé, les SNS étaient exposés à des menaces de désacralisation et par conséquent de disparition.

Figure 6 : Caractérisation des pressions subies par les SNS

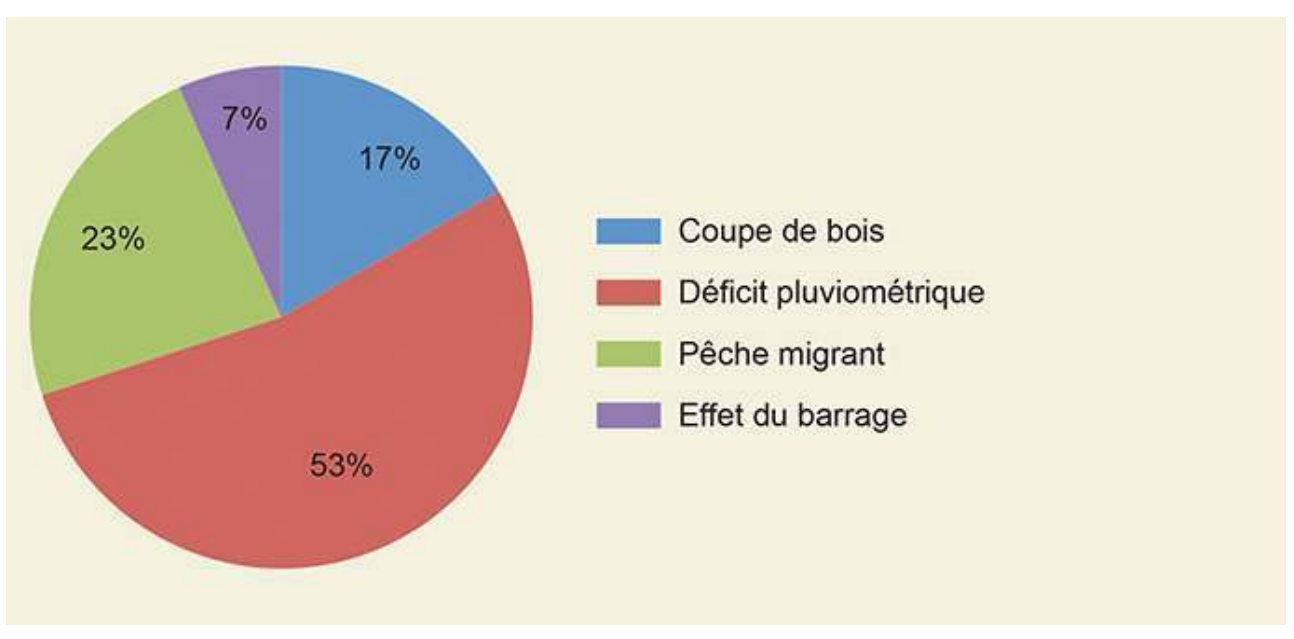

Source : Enquêtes de terrain 2011 
Figure 7 : État actuel de conservation du couvert végétal des SNS étudiés

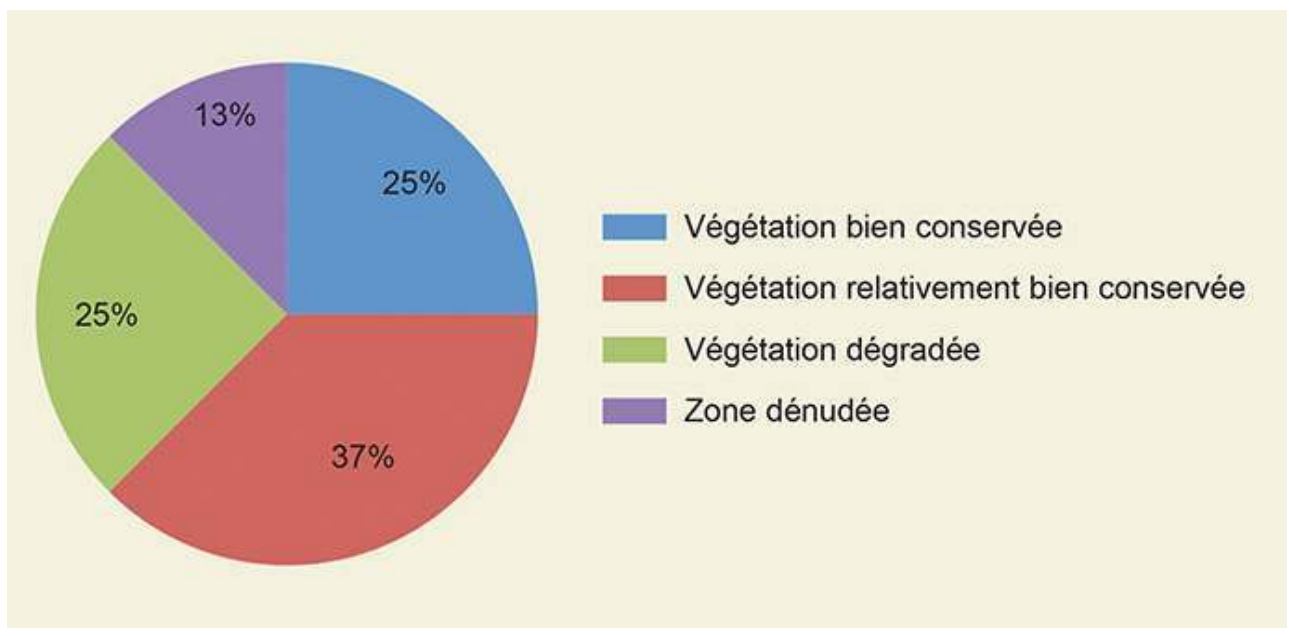

Source : Enquêtes de terrain 2011

Aujourd'hui, avec la mise en place de l'APAC, le rôle et l'importance traditionnelle du SNS de Mitij ont été revalorisés dans le système de gestion de l'APAC, contribuant à sa protection. Parallèlement, le dispositif de protection des sites de Boukinoum fouloup, de Alafouloup, de Koutéwol, et de Kiling-kiling a été renforcé. Même si les règles traditionnelles et les interdits sont encore très respectés dans la plupart des sites situés en dehors des AP reconnues officiellement, comme c'est le cas dans le Bliss-Kassa, ces derniers restent vulnérables, car les pressions demeurent.

\section{Rôle socio-culturel et écologique des sites naturels sacrés}

Les sites naturels sacrés sont le centre de pratiques et de valeurs traditionnelles pour les populations locales, qui de ce fait abritent une biodiversité non négligeable. Ce sont des lieux particulièrement respectés et protégés, car symbolisant des valeurs culturelles et spirituelles pour ces populations. Les SNS marins et côtiers sont des lieux importants pour les habitants des villages riverains. Ce sont des sites entourés de mystères, à l'intérieur desquels les populations locales protègent d'une part certaines espèces aquatiques et terrestres considérées comme leurs doubles (totems) et réglementent l'accès à et l'exploitation de certaines ressources naturelles stratégiques d'autre part, au travers de l'implantation d'un fétiche par exemple. La grande majorité de ces sites préserve également la mangrove qui sert d'habitat à plusieurs espèces marines. Dans la commune de Mangagoulack les villageois s'étant rendu compte de la disparition progressive des ressources halieutiques dans les eaux de l'estuaire qui autrefois était naturellement très riches, ont décidé de prendre les choses en main pour remédier à cette situation. C'est ainsi qu'ils ont eu l'idée de réhabiliter les anciennes règles traditionnelles qui permettaient la préservation des ressources halieutiques. Le fétiche qui fait respecter ces règles a été implanté au niveau des SNS de Mitij et de Kiling-kiling en vue de restaurer les fonctions importantes (zone de reproduction, de nurserie et de repos des espèces halieutiques) que ces sites remplissaient traditionnellement.

Par les cultes qui s'y tiennent et les divers interdits qui les entourent, les espaces sacrés sont préservés et les ressources naturelles (animales et végétales) localisées dans ces lieux bénéficient ainsi de cette protection. Les systèmes de gestion traditionnels mis en place

Revue d'ethnoécologie, 11 | 2017 
visent d'abord à préserver ces lieux d'importance culturelle, l'harmonie avec les ancêtres, les génies et les divinités. Ainsi, nous conviendrons avec Dugast (2002) que :

«derrière toutes ces pratiques, c'est bien le souci d'un contrôle de force contribuant à assurer la subsistance du groupe que l'on décèle, et non la préoccupation de préserver l'environnement ».

Bien que n'ayant pas pour premier objectif la protection de l'environnement, ces SNS contribuent indirectement à la conservation des ressources naturelles animales et végétales, car leurs fonctions font qu'ils ont un impact positif sur l'environnement.

Par conséquent, ces méthodes de gestion traditionnelle, qui jadis faisaient office de réglementation gagneraient à être prises en compte de manière officielle pour une meilleure gestion des ressources naturelles au Sénégal. À ce sujet Roué (2012) est d'avis que

« dans certains cas pourtant, des programmes de cogestion permettent à un État ou une administration centrale de reconnaître la légitimité des droits des populations autochtones, en particulier celle de gérer les terres qui sont les leurs ».

Ces sites constituent de véritables lieux de préservation des secrets et pratiques multiséculaires. Une forte communion existe entre les populations et les esprits ou êtres qui résident dans ces endroits. Des offrandes et des sacrifices sont effectués pour la conservation des sites et de leurs occupants. C'est dans cette logique que les valeurs d'identité culturelle et spirituelle ainsi que l'organisation sociale de la population se trouvent étroitement liées à la nature, aux ressources naturelles en général. Dès lors, l'ensemble des interdits et des dissuasions mystiques qui entourent ces sites participent à leur conservation. Ces facteurs socioculturels influencent la sauvegarde de la biodiversité (Sow 2003).

Cette contribution des SNS et d'autres mesures de conservation locales efficaces pour le maintien de la biodiversité et le besoin de mieux reconnaitre et valoriser ces bénéfices, tout en préservant et en renforçant leur vocation primaire, font l'objet d'une attention croissante au niveau international ${ }^{10}$.

\section{Conclusion}

47 La vocation primaire des sites naturels sacrés réside dans le maintien de l'identité culturelle du peuple diola. Les SNS du Bliss-Kassa et de l'APAC de Mangagoulack remplissent ainsi des fonctions sociales et religieuses, mais également écologiques importantes. Beaucoup de sites naturels marins et terrestres (îles, bras de mer, forêts, etc.) sont considérés comme sacrés ou gnei-gnei (en Diola). Ces espaces sacrés sont d'une grande valeur culturelle qui préserve les secrets et pratiques multiséculaires des Diolas. Ils abritent les mânes des ancêtres et d'autres divinités qui assurent un lien intrinsèque avec les populations. Divers types de SNS sont rencontrés. Ils se distinguent les uns des autres par leur objectif et par les règles spécifiques qui les régissent. Toutefois, les règles régissant les SNS abritant des totems ou un fétiche demeurent plus contraignantes.

Les règles de gestion qui entourent les SNS ont des bénéfices écologiques remarquables. Dans certaines zones, comme le Bliss-Kassa, les SNS conservent toujours les mêmes interdits malgré l'influence des religions dites révélées et la modernisation. Signalons que les Diola du Bliss-Kassa sont originaires du Kassa (Kassa correspond au département 
d'Oussouye), et sont considérés comme un des groupes qui ont su garder farouchement leurs valeurs culturelles (Badiane 2006).

L'importance des SNS dans la conservation des écosystèmes et de la diversité biologique est appréhendée ici par le biais de la richesse spécifique de ces lieux, qui fait d'eux de véritables sanctuaires de biodiversité. La préservation de la sacralité est importante pour le maintien des valeurs socioculturelles. De plus si elle était reconnue et valorisée de manière adéquate, elle contribuerait clairement à la gestion des ressources naturelles. Cela implique cependant une démarche passant par la reconnaissance et la préservation des SNS et des droits de gestion traditionnelle par les communautés locales dans les systèmes de protection conventionnels.

À l'heure actuelle, au Sénégal, les lois et règlements en vigueur (code forestier, de la chasse et de la pêche) n'abordent pas de façon claire et précise les savoirs endogènes, normes et pratiques culturelles. Les SNS ne sont donc pas reconnus et ne bénéficient pas d'un cadre juridique de protection, ni de soutien des programmes gouvernementaux et restent exposés à des menaces importantes. Vu leur rôle et leur importance, il serait souhaitable de promouvoir les SNS côtiers et marins de la région casamançaise et leurs valeurs socioculturelles ainsi que leurs bénéfices pour la préservation des ressources naturelles. La mise en place des lois et des règlementations plus spécifiques pour les SNS en vue de leur reconnaissance formelle renforcerait les mécanismes et institutions de gestion des communautés locales, et les protègerait face aux pressions extérieures à l'intérieur comme à l'extérieur des AP reconnues officiellement.

CBD 2016. Recommandations adoptées par l'organe subsidiaire chargé de fournir des avis scientifiques, techniques et technologiques. https://www.cbd.int/doc/recommendations/ sbstta-20/sbstta-20-rec-01-fr.pdf

\section{BIBLIOGRAPHIE}

Badiane S.D. 2006 - Les pratiques culturelles et gestion des ressources forestières en basse Casamance: l'exemple des Diola d'Oussouye. Mémoire de DEA, Géographie, option : environnement, Université Gaston Berger Saint-Louis, Sénégal, 83 p.

Badiane S.D. 2012 - Espaces forestiers de la Casamance amphibie (Sénégal). Déterminisme territorial, biodiversité et stratégies de conservation. Thèse de doctorat unique. Spécialité: Environnement littoral, Université Cheikh Anta Diop, Dakar, 320 p.

Borrini-Feyerabend G., Chatelain C. \& Tous P. 2009 - Rapport pour l'Association des Pêcheurs de la Communauté Rurale de Mangagoulack. CENESTA, PNUD/FEM/SGP, FIBA, 79 p.

Butare I. 2003 - Pratiques culturelles, la sauvegarde et la conservation de la biodiversité en Afrique de l'Ouest et du centre: Actes du Séminaire-Atelier de Ouagadougou (Burkina Faso) du 18 au 21 juin 2001. Ottawa, IDRC/CRDI, Zoom Éditions, 251 p.

Chabi-Yaouré F. 2009 - Caractérisation des savoirs locaux et des modes traditionnels de gestion des ressources marines et côtières dans les futures AMP du Petit Kassa et de Pt Saint Georges en Casamance 
(Sénégal). Mémoire de fin d'étude master spécialisé en gestion des aires protégées. Dakar, Institut International des Sciences de l'Ingénierie de l'Eau et de l'Environnement, 73 p.

Diatta C.S. 2012 - Le rôle des sites naturels sacrés pour la conservation des ressources naturelles marines et côtières : l'exemple du Petit Kassa et de l'aire du patrimoine autochtone et communautaire de Mangagoulack (Casamance). Mémoire de Master2, Institut Universitaire des Pêches et d'Aquaculture (IUPA), $87 \mathrm{p}$.

Diedhiou P. 2011 - L’identité Jóola en question (Casamance). La bataille idéologique du MFDC pour l'indépendance. Paris, Karthala, $404 \mathrm{p}$.

Dieme I.Y. 2008 - Contribution au processus de mise en place de l'AMP du Petit Kassa. Aspects socioéconomiques. Mémoire de DESS. IUPA, Université Cheikh Anta Diop, 57 p.

Dieng S. 2009 - Sur les traces d'El-Hadj Omar. Regards croisés sur l'homme et l'œuvre. Dakar, Les nouvelles Éditions Africaines du Sénégal, 220 p.

Direction de la Prévision et de la Statistique 2002 - Recensement Général de la Population et de l'Habitat : Répertoire des villages de la région de Ziguinchor. Dakar, Direction de la Prévision et de la Statistique, $64 \mathrm{p}$.

Djoghlaf A. 2006 - Intervention au symposium Diversité culturelle, identités et mondialisation organisé par l'Association internationale de science politique (AISP), Montréal, le 12 octobre 2006.

Dugast S. 2002 : Modes d'appréhension de la nature et gestion patrimoniale du milieu, in: Cormier-Salem M.C. et al. Patrimonialiser la nature tropicale. Dynamiques locales et enjeux internationaux : 31-78.

Dumez R. et Ka M. (2000) : « TERRITOIRE ASSIÉGÉ YOFF. Un village lébou dans la banlieue de Dakar ». Collection Dossiers régions côtières et petites îles 7. Ed. UNESCO 2000.CNRS - Programme Environnement Université Cheikh Anta Diop de Dakar - Chaire UNESCO. 81 pages. Site web: http://www.unesco.org/csi/pub/papers2/yoff.htm.

Dupire M. (1991) : «: Totems sereer et contrôle rituel de l'environnement ». In: L'Homme, 1991, tome 31 n$^{\circ} 118$, p. 37-66. http://www.persee.fr/doc/hom_0439-4216_1991_num_31_118_369379

Fall S.M., Diouf M. \& Badiane S.D. 2010 - Identification et caractérisation des sites naturels sacrés côtiers et marins en Afrique de l'ouest. Rapport du Sénégal, FIBA, RAMPAO, PCRM. 52 p.

Gadou D.M. 2003 - Préservation de la biodiversité: les réponses des religions africaines. In Butare I. Pratiques culturelles et conservation de la biodiversité en Afrique de l'Ouest et du centre». IDRC/CRDI. Zoom Edition : 47-68.

Groupe OCC (Oumar Cissé et Compagnie) 2010a - Plan Local de Développement (PLD), Communauté rurale de Kafountine, $94 \mathrm{p}$.

Groupe OCC (Oumar Cissé et Compagnie) 2010b - Plan Local de Développement (PLD), Communauté rurale de Mangagoulack $97 \mathrm{p}$.

Journet 0. 1976 - Rôles et statuts des femmes dans la société diola de Basse Casamance. Thèse de Doctorat de 3ème cycle, Université de Lyon 521 p.

Journet 0. 1979 - Questions à propos du sacrifice chez les Diola de Basse-Casamance. Systèmes de Pensée en Afrique Noire - Le sacrifice III : 77-94. http://span.revues.org/418

Journet-Diallo 0. 1998 -Un monde diffracté. Théories joola du double animal. Systèmes de pensée en Afrique noire : 203-230. 
Juhé-Beaulaton D. 2006 - Les sites sacrés naturels et la conservation de la biodiversité. Enjeux politiques, économiques et sociaux (études de cas au Bénin, Burkina Faso et Togo). «Des bois sacrés vodou aux paysages culturels : la conservation de la biodiversité en question (Sud Togo et Bénin) ». Convention INRA /PARIS. RAPPORT FINAL, $47 \mathrm{p}$.

Juhé-Beaulaton D. 2008 - Sacred forests and the global challenge of biodiversity conservation: the case of Benin and Togo. Journal for the Study of Religion, Nature, and Culture : 351-372. https:// hal.archives-ouvertes.fr/halshs-00090462/document

Kante B. 2012 - Appui juridique sur le statut légal des projets d'APAC de Petit Kassa et de Mlomp. Rapport de mission/FIBA, $31 \mathrm{p}$.

Ndiaye P. \& Diagne M. 2004 - Étude des Sites sacrés de la Presqu'île du Cap-Vert. État de Référence: Connaissances sur les Sites sacrés de la Presqu'île du Cap-Vert Inventaire et classement des Sites sacrés. Mise en place du Comité de Gestion des Écosystèmes Marins de la Presqu'île du Cap-Vert (COGEM Cap-Vert), $68 \mathrm{p}$.

Oyono P.R., Fall S.M., Karibuhoye C.S., Said A.R., Sidibeh L. \& Sow M. 2012 - Les Sites Naturels Sacrés de l'Écorégion Côtière et Marine Ouest-Africaine. Une Exploration et des Options de Reconnaissance Institutionnelle. Dakar, FIBA, $49 \mathrm{p}$.

Palmeri P. 1995 - Retour dans un Village Diola de Casamance. Chronique d'une recherche anthropologique au Sénégal. Paris, L'Harmattan, 397 p.

Pélissier P. 1966 - Les paysans du Sénégal. Les civilisations agraires du Cayor à la Casamance. Fabrègue St Yrieux, $939 \mathrm{p}$.

Petitjean P., Zharov V., Glaser G., Richardson J., Padirac B. de \& Archibald G. (Ed.) 2009 - Soixante ans de science à l'UNESCO, 1945-2005. Paris, UNESCO, 780 p.

Roué M. 2012 - Histoire et épistémologie des savoirs locaux et autochtones De la tradition à la mode. Revue d'ethnoécologie 1. http://ethnoecologie.revues.org/813 DOI : 10.4000/

ethnoecologie. 813

Sagna P. 2007 - Caractéristiques climatiques, in Atlas du Sénégal, 1e édition, Paris, Les Éditions J.A. aux éditions Jaguar : 66-69.

Snyder F.G. 1973 - L'évolution du droit foncier diola de Basse-Casamance (Republique du Sénégal) : étude d'anthropologie juridique des rapports entre les hommes et les terres chez les Diola-Bandial. Thèse de Doctorat, Paris, Université Paris 1 Panthéon Sorbonne, 550 p.

Sow M. 2003 - Rôle des structures traditionnelles dans la valorisation de la biodiversité, In Butare I. Pratiques culturelles et conservation de la biodiversité en Afrique de l'Ouest et du centre. IDRC/CRDI, Zoom Edition : 205-212.

Taïqui L., Bensalah H. \& Seva E. 2009 - La conservation des sites naturels sacrés au Maroc : est-elle compatible avec le développement socio-économique? Mediterranea serie de Estudios Biológicos, Ėpoca II, 20, Universidad de Alicante, 46 p.

Thomas L.V. 1958 - Les Diola : essais d'analyse fonctionnelle sur une population de Basse Casamance. Mémoires IFAN-Dakar 55, 343 p.

Thomas L.V. 1959 - Les Diola, essai d'analyse fonctionnelle sur une population de Basse Casamance. Mémoire IFAN 55, $821 \mathrm{p}$.

UICN 2012 - http://2012congress.iucn.org/fr/assemblee_des_membres/resolutions/ 


\section{ANNEXES}

\section{1 - Caractéristiques des sites naturels sacrés}

\begin{tabular}{|c|c|c|c|c|c|c|}
\hline \multirow{2}{*}{\multicolumn{2}{|c|}{$\begin{array}{l}\text { Villages de localisation des SNS } \\
\text { Nom des SNS }\end{array}$}} & \multicolumn{2}{|c|}{ Bakassouk } & \multicolumn{3}{|c|}{ Hitou } \\
\hline & & Efahay yata Salindia & Guéfouté & \multicolumn{2}{|l|}{ Sankikitora } & Koutène \\
\hline \multirow{4}{*}{ Raison sacralité } & Totem & & & & & \\
\hline & Fétiche & & & & & \\
\hline & Lieu de culte & & & & & \\
\hline & Génie et Fétiche & & & & & \\
\hline \multicolumn{2}{|l|}{ Espèces totémiques } & Baleine & $\begin{array}{c}\text { Poisson génie } \\
\text { (sous la forme d'une sirène) }\end{array}$ & \multicolumn{2}{|l|}{ Requin } & Crocodile \\
\hline \multicolumn{2}{|c|}{ Nom local des espèces totémiques } & $\begin{array}{c}\text { Effot (Kassa)/ } \\
\text { Erounghalène (Blouf) }\end{array}$ & Ingnankanpadj & \multicolumn{2}{|c|}{ Hougnongh (Kassa) } & Yone \\
\hline \multicolumn{2}{|l|}{ Famille dépositaire } & $\begin{array}{l}\text { DIASSY et DEMBA } \\
\text { de Diowote }\end{array}$ & Familles du village & \multicolumn{2}{|c|}{$\begin{array}{l}\text { DIEME d'Erindiagne de Hitou et } \\
\text { DIEME d'Esangnoul de Niomoune }\end{array}$} & DIATTA de Diammo \\
\hline \multirow[b]{2}{*}{ Type de protection } & Traditionnel & $\mathrm{x}$ & $\mathrm{x}$ & & & $\mathrm{x}$ \\
\hline & $\begin{array}{l}\text { Moderne } \\
\text { (Aire Protégée) }\end{array}$ & $\mathrm{x}$ & & & & \\
\hline \multirow{3}{*}{ Interdit } & Total & $\mathrm{x}$ & $\mathrm{x}$ & & & $\mathrm{x}$ \\
\hline & Partiel & & & & & \\
\hline & Aucun & & & & & \\
\hline \multirow{3}{*}{ Ressources exploitées } & Mangrove & & & & & \\
\hline & Poisson & & & & & \\
\hline & Coquillage & & & & & \\
\hline \multicolumn{2}{|c|}{ Personnes autorisées à accéder aux SNS } & Aucune & Aucun prélèvement & Résidents locaux & & Aucune \\
\hline \multicolumn{2}{|c|}{ Exploitation des ressources } & Aucune & Aucun prélèvement & Récolte de coquillage un & niquement & Aucun prélèvement \\
\hline & Totem & Lieu de $\mathrm{C}$ & Fétiche & Gé & énie et Fétic & \\
\hline \multicolumn{2}{|c|}{ Villages de localisation des SNS } & \multicolumn{2}{|c|}{ Niomoune } & \multicolumn{3}{|c|}{ Mangagoulack } \\
\hline Nom des SNS & & Gnangabitou & Diahébène & Mitij & & Koutéwol \\
\hline \multirow{4}{*}{ Raison sacralité } & Totem & & & & & \\
\hline & Fétiche & & & & & \\
\hline & Lieu de culte & & & & & \\
\hline & Génie et Fétiche & & & & & \\
\hline \multicolumn{2}{|l|}{ Espèces totémiques } & Requin marteau & Requin marteau & -क्-m & & Hippopotame \\
\hline \multicolumn{2}{|c|}{ Nom local des espèces totémiques } & Hougnongh (Kassa) & Hougnongh (Kassa) & 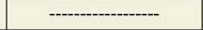 & & Ekaw (Blouf) \\
\hline \multicolumn{2}{|l|}{ Famille dépositaire } & BADJI d'Oubak & $\begin{array}{l}\text { DIATTA d'Esangnoul } \\
\text { Kabounkoute }\end{array}$ & $\begin{array}{c}\text { Aucune famille } \\
\text { (SNS communautaire) }\end{array}$ & DIAT & TA d'Oulempane \\
\hline \multirow[b]{2}{*}{ Type de protection } & Traditionnel & $\mathrm{x}$ & $\mathrm{x}$ & $\mathrm{x}$ & & $x$ \\
\hline & $\begin{array}{l}\text { Moderne } \\
\text { (Aire Protégée) }\end{array}$ & & & $\mathrm{x}$ & & $x$ \\
\hline \multirow{3}{*}{ Interdit } & Total & $x$ & $\mathrm{x}$ & $x$ & & \\
\hline & Partiel & & & & & \\
\hline & Aucun & & & & & $\mathrm{x}$ \\
\hline \multirow{3}{*}{ Ressources exploitées } & Mangrove & & & & & \\
\hline & Poisson & & & & & $x$ \\
\hline & Coquillage & & & & & $\mathrm{x}$ \\
\hline Personnes autorisées à & accéder aux SNS & Aucune & Aucune & Aucune & & sidents locaux \\
\hline Exploitation des ressou & urces & Aucun prélèvement & Aucun prélèvement & Aucun prélèvement & $\begin{array}{r}\text { Unique } \\
\text { ressortiss } \\
\text { de }\end{array}$ & $\begin{array}{l}\text { ement permis aux } \\
\text { sants de la commune } \\
\text { Mangagoulack }\end{array}$ \\
\hline
\end{tabular}




\begin{tabular}{|c|c|c|c|c|c|}
\hline \multirow{2}{*}{\multicolumn{2}{|c|}{$\begin{array}{l}\text { Villages de localisation des SNS } \\
\text { Nom des SNS }\end{array}$}} & \multirow{2}{*}{$\begin{array}{c}\text { Tendouck } \\
\text { Boukinoum fouloup }\end{array}$} & \multirow{2}{*}{$\begin{array}{c}\text { Boutégol } \\
\text { Alafouloup }\end{array}$} & \multicolumn{2}{|c|}{ Boutème } \\
\hline & & & & Brélosso & Ecobe ou Kiling-kiling \\
\hline \multirow{4}{*}{ Raison sacralité } & Totem & & & & \\
\hline & Fétiche & & & & \\
\hline & Lieu de culte & & & & \\
\hline & Génie et Fétiche & & & & \\
\hline \multicolumn{2}{|l|}{ Espèces totémiques } & Lamantin & Requin & Requin & - \\
\hline \multicolumn{2}{|c|}{ Nom local des espèces totémiques } & Balirail (Blouf) & Fougnone (Blouf) & Fougnone (Blouf) & -..-- \\
\hline \multicolumn{2}{|l|}{ Famille dépositaire } & $\begin{array}{l}\text { DIEDHIOU } \\
\text { de Bourombone }\end{array}$ & $\begin{array}{l}\text { DIATTA d'Enébane } \\
\text { de Tendouck }\end{array}$ & $\begin{array}{l}\text { SAMBOU } \\
\text { de Bouguanefou }\end{array}$ & $\begin{array}{l}\text { Aucune famille } \\
\text { (SNS communautaire) }\end{array}$ \\
\hline \multirow[b]{2}{*}{ Type de protection } & Traditionnel & $\mathrm{x}$ & $\mathrm{x}$ & & $\mathrm{x}$ \\
\hline & $\begin{array}{l}\text { Moderne } \\
\text { (Aire Protégée) }\end{array}$ & $\mathrm{x}$ & $\mathrm{x}$ & & \\
\hline \multirow{3}{*}{ Interdit } & Total & & $\mathrm{x}$ & & $\mathrm{x}$ \\
\hline & Partiel & $\mathrm{x}$ & & & \\
\hline & Aucun & & & $\mathrm{x}$ & \\
\hline \multirow{3}{*}{ Ressources exploitées } & Mangrove & & & & \\
\hline & Poisson & & & $\mathrm{x}$ & \\
\hline & Coquillage & & & $\mathrm{x}$ & \\
\hline \multicolumn{2}{|c|}{ Personnes autorisées à accéder aux SNS } & Famille dépositaire & Aucune & N'importe qui & Aucune \\
\hline \multicolumn{2}{|c|}{ Exploitation des ressources } & Famille dépositaire & Aucun prélèvement & Libre & Aucun prélèvement \\
\hline & ] Totem & ] Lieu de culte & Fétiche & Génie & Fétiche \\
\hline \multicolumn{2}{|c|}{ Villages de localisation des SNS } & \multicolumn{2}{|c|}{ Affignam } & \multicolumn{2}{|c|}{ Elana } \\
\hline \multicolumn{2}{|l|}{ Nom des SNS } & Guagoulok & Yaboufassor & Foumor sithégour & Barotor \\
\hline \multirow{4}{*}{ Raison sacralité } & Totem & & & & \\
\hline & Fétiche & & & & \\
\hline & Lieu de culte & & & & \\
\hline & Génie et Fétiche & & & & \\
\hline \multicolumn{2}{|l|}{ Espèces totémiques } & - & Requin & Requin & -1-5 \\
\hline \multicolumn{2}{|c|}{ Nom local des espèces totémiques } & - & Fougnone (Blouf) & Fougnone (Blouf) & - \\
\hline \multicolumn{2}{|l|}{ Famille dépositaire } & COLY du village & DIATTA de Djiloguir & SAGNA de Kagoute & DIATTA de Kalé \\
\hline \multirow[b]{2}{*}{ Type de protection } & Traditionnel & $\mathrm{x}$ & $\mathrm{x}$ & & $\mathrm{x}$ \\
\hline & $\begin{array}{l}\text { Moderne } \\
\text { (Aire Protégée) }\end{array}$ & & & $\mathrm{x}$ & \\
\hline \multirow{3}{*}{ Interdit } & Total & & & & \\
\hline & Partiel & & & & \\
\hline & Aucun & $\mathrm{x}$ & $\mathrm{x}$ & $\mathrm{x}$ & $\mathrm{x}$ \\
\hline \multirow{3}{*}{ Ressources exploitées } & Mangrove & $\mathrm{x}$ & $\mathrm{x}$ & $\mathrm{x}$ & \multirow{3}{*}{ Pas de ressource exploitée } \\
\hline & Poisson & & & & \\
\hline & Coquillage & $\mathrm{x}$ & $\mathrm{x}$ & $x$ & \\
\hline \multicolumn{2}{|c|}{ Personnes autorisées à accéder aux SNS } & N'importe qui & N'importe qui & Résidents locaux & N'importe qui \\
\hline Exploitation des ressol & urces & Libre & Libre & $\begin{array}{l}\text { Uniquement permis } \\
\text { aux ressortissants de la } \\
\text { commune de } \\
\text { Mangagoulack }\end{array}$ & Libre \\
\hline & Totem & Lieu de & & & hie et Fétiche \\
\hline
\end{tabular}

Source : Enquêtes de terrain 2011

\section{2 - État actuel de conservation du couvert végétal des SNS étudiés}




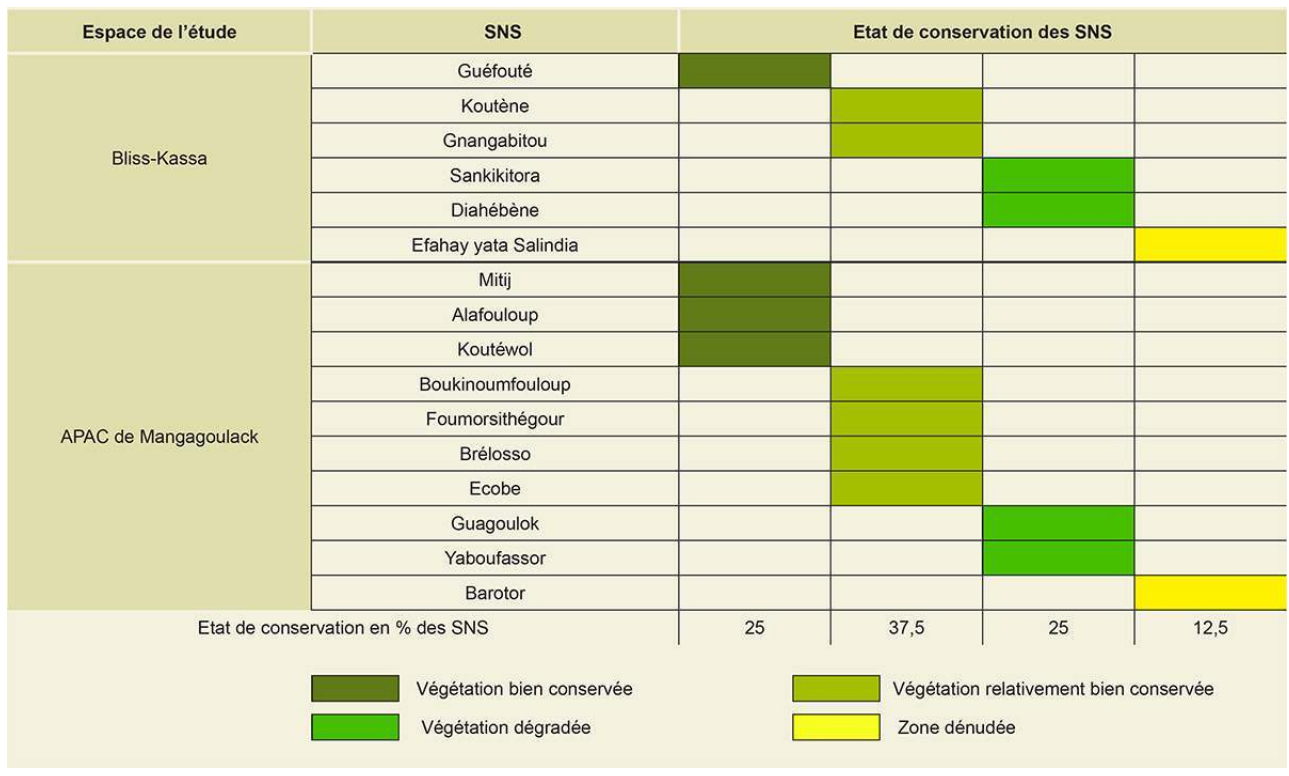

Source : Enquêtes de terrain 2011

\section{NOTES}

1. Le Petit Kassa est désigné aujourd'hui sous l'appellation de Bliss-Kassa.

2. Les Diolas représentent l'ethnie majoritaire en Basse Casamance et leur territoire s'étend du sud de la Gambie au nord de la Guinée Bissau. L'appellation de diola viendrait des mandings, signifiant "peuples du littoral ou ceux qui aiment se faire justice ». Mais, le véritable nom de cette ethnie est «Ajamat» qui signifie " ceux qui sont solidaires ». Le terme diola s'est donc imposé à l'ensemble des entités de ce groupe du fait que les populations ajamat ont connu un exode massif vers le Sénégal pendant la période coloniale (Diedhiou 2011).

3. Lexique de quelques SNS : Efahay yata Salindia : le banc de sable de Salindia, Boukinoum fouloup : habitat des fouloup, Foumor sithégour : dortoir des perroquets, Barotor : la source, Brélosso : lieu de rencontre, Kiling-kiling : un par un, Yaboufassor : lieu de dispersement.

4. Mot manding qui veut dire « un par un ».

5. El Hadji Omar apparaît comme l'une des plus fortes personnalités de la culture arabo-islamique en Afrique au sud du Sahara. Il naquit à Halwâr, un village situé à quelques dizaines de kilomètres de Podor, entre 1794 et 1797 . Vers 1827, il partit à la Mecque où il resta plusieurs années. À son retour il s'installa à Dinguiraye avec l'intention de faire la guerre sainte en passant du BasSénégal au Haut-Sénégal (Dieng 2009).

6. Les Felup ou Floup sont les gens habitant dans la région d'Oussouye, dans la partie méridionale de la Casamance (Pélissier 1966).

7. Eloenkin est un boekin de cohésion sociale. Il est le centre de gravité des deux ordres initiatiques à Oussouye.

8. Ehuña abrite le fétiche d'initiation à la société des femmes. Ces deux derniers sites terrestres ne font pas partie des 16 SNS étudiés.

9. Le SNS de Efahay yata Salindia est une langue de terre sableuse sans aucune végétation qui constitue un site de reproduction par excellence et un dortoir d'oiseaux. Il fait partie du grand ensemble connu sous le nom de presqu'île aux oiseaux.

10. Voir notamment les résolutions du congrès mondial de l'UICN de Jeju en 2012 et les recommandations de l'organe subsidiaire consultatif scientifique et technique (SBSTTA) de la CDB en 2016. 


\section{RÉSUMÉS}

Les sites naturels sacrés (SNS) ont une importance culturelle, sociale et spirituelle pour les populations diola de la Basse Casamance (sud du Sénégal). De plus, ils remplissent une fonction vitale pour la conservation des ressources naturelles et la biodiversité en général et présentent ainsi un intérêt écologique certain. Dans le Bliss-Kassa et dans l'Aire du Patrimoine Autochtone et Communautaire de Mangagoulack, les SNS sont encore très présents. Les populations diola entretiennent des relations étroites avec la nature dont certains éléments sont considérés comme faisant partie intégrante de la société. Quelques sites naturels sacrés ont fait l'objet d'une recherche en vue d'une meilleure connaissance de leur fonctionnement, mais aussi pour mettre en lumière leur contribution à la conservation durable des ressources marines et côtières.

L'article met en évidence le rôle des sites naturels sacrés dans la conservation des ressources naturelles marines et côtières hier et aujourd'hui, les méthodes traditionnelles de gouvernance et de conservation de ces sites, leur état de conservation, et les menaces auxquelles ils sont exposés.

Sacred and natural sites have a social, cultural and spiritual importance for the Jola people in Lower Casamance. Moreover, they have a vital role for the preservation of natural resources and the biodiversity in general and then, undoubtedly present an ecological interest. In the BlissKassa and in the Community Conserved Area of Mangagoulack, SNS are still very present. The Jola people have very close relations with nature, some of which elements are considered as parts and parcel of their society. Some sacred natural sites were included in this research with a view to a better understanding of their functioning, but also to shed light on how they contribute to the lasting preservation of marine and coastal resources.

This paper highlights the role of sacred and natural sites for the preservation of natural, sea and coastal resources in the past and today, the traditional management and preservation methods of those sites, their state of conservation and the threats they are facing.

\section{INDEX}

Index géographique : Sénégal, Casamance

Mots-clés : sites naturels sacrés, conservation, ressources marines et côtières, Bliss-Kassa, Aire du Patrimoine Autochtone et Communautaire, Mangagoulack

Keywords : sacred natural sites, preservation, marine and coastal resources, Bliss-Kassa, Community Conserved Area, Mangagoulack

\section{AUTEURS}

\section{CLAUDETTE SOUMBANE DIATTA}

Département de Géographie, Faculté des Sciences Humaines, Université Cheikh Anta Diop, Dakar Sénégal 


\section{MALICK DIOUF}

Département de Biologie Animale, Faculté des Sciences et Techniques, Institut Universitaire de pêche et d'aquaculture, Université Cheikh Anta Diop, Dakar (Sénégal)

\section{CHARLOTTE KARIBUHOYE}

Fondation pour la Nature (MAVA)

\section{AMADOU ABDOUL SOW}

Département de Géographie, Faculté des Sciences Humaines, Université Cheikh Anta Diop, Dakar (Sénégal) 